12

\title{
Дальнейшее развитие и уточнение концепции эффективного потенциала для стробоскопических выборок координат и скоростей ионов в квадрупольных радиочастотных полях
}

\author{
(C) А.С. Бердников, Н.Р. Галль, С.В. Масюкевич
}

Институт аналитического приборостроения РАН, 190103 Санкт-Петербург, Россия

e-mail: asberd@yandex.ru

Поступило в Редакцию 1 ноября 2018 г.

В окончательной редакции 9 января 2019 г.

Принято к публикации 10 января 2019 г.

\begin{abstract}
Рассмотрена недавно предложенная концепция эффективного потенциала, разработанная на основе анализа стробоскопических выборок координат и скоростей ионов при их движении в радиочастотном квадрупольном поле. Получены точные формулы для стробоскопической модели движения ионов, справедливые в любой точке зоны устойчивости. Анализ полученных формул привел к корректировке некоторых положений исходной концепции эффективного потенциала стробоскопических выборок, разработанной для окрестности вершины зоны устойчивости.
\end{abstract}

DOI: 10.21883/JTF.2019.07.47808.383-18

Техника масс-селективной фильтрации заряженных частиц в радиочастотных электрических полях, открытая В. Паулем [1], является основой для работы радиочастотных ионных ловушек и квадрупольных фильтров масс [2-7]. Фильтрация ионов с определенными массами в этих устройствах, использующих синусоидальные радиочастотные напряжения, основана на свойствах уравнения Матье [8], с помощью которого описывается движение ионов в радиочастотных квадрупольных полях. Ионные ловушки и квадрупольные фильтры масс, которые используют данный эффект, получили широкое практическое применение в качестве недорогих, компактных и надежно работающих в самых разных условиях масс-анализаторов с умеренными требованиями к разрешающей способности и диапазону масс [2-7]. Однако строгая математическая теория решений уравнения Матье не обладает необходимой наглядностью, с помощью которой можно было бы легко синтезировать радиочастотные масс-фильтры с требуемыми характеристиками.

Псевдопотенциальная модель [9], описывающая динамику движения точечной массы под воздействием быстро осциллирующей потенциальной силы, обеспечивает наглядное качественное описание движения ионов в радиочастотных электрических полях $[10,11]$. Однако для задач, связанных с анализом движения ионов в радиочастотных квадруполях (квадрупольных фильтрах масс, квадрупольных радиочастотных ловушках) [2-7], это качественное описание является недостаточно точным. Имеется объективная потребность в создании уточненной теории движения ионов в квадрупольных радиочастотных электрических полях, которая бы соединяла наглядность псевдопотенциальной модели с улучшенной точностью описания (в идеале - с абсолютно точным описанием) поведения ионов. Число таких попыток, предпринятых в последнее время, достаточно велико [12-20].

Настоящая работа посвящена уточнению результатов публикации [14] и их распространению на все точки первой зоны устойчивости радиочастотного квадруполя вместо окрестности вершины зоны устойчивости. В частности, будет показано, что в любой точке зоны устойчивости стробоскопические выборки координат и скоростей при движении ионов в радиочастотном квадрупольном поле описываются секулярными колебаниями с безразмерной частотой $\beta$ или в качестве альтернативного способа описания биениями с безразмерной частотой $1-\beta$ (где $\beta-$ параметр Флоке [21-23]). Кроме того, на основе предлагаемой теории для окрестности вершины зоны устойчивости будут получены приближенные аналитические оценки для границ зоны и для глубины и ширины псевдопотенциальных ям, характеризующих аксептанс квадрупольного масс-анализатора. Использование этих выражений значительно упрощает анализ и оптимизацию режимов работы квадрупольных масс-анализаторов и квадрупольных масс-фильтров.

Предметом исследования являются траектории $x(t), y(t) \quad$ и скорости $\quad v_{x}(t)=\dot{x}(t), \quad v_{y}(t)=\dot{y}(t) \quad$ при движении ионов в радиочастотном поле линейного квадрупольного масс-фильтра с электрическим потенциалом

$$
U(x, y, t)=\left(U_{0}+V_{0} F(t)\right)\left(x^{2}-y^{2}\right) / r_{0}^{2},
$$

где $x, y$ - декартовы координаты, $r_{0}$ - радиус апертуры между гиперболическими электродами линейного квадруполя, $U_{0}$ - постоянная компонента напряжения, $V_{0}$ - амплитуда радиочастотной компоненты напряжения, $F(t)$ - периодическая функция с нулевым средним и периодом $T=2 \pi / \Omega$ для радиочастотной составляю- 
щей напряжения, $\Omega$ - базовая круговая частота радиочастотной составляющей, $t-$ время. Для частного случая линейного квадруполя с косинусоидальными напряжениями $F(t)=\cos \left(\Omega t+\varphi_{0}\right)$, где $\varphi_{0}-$ фаза радиочастотного напряжения в момент старта иона $t=t_{0}=0$.

Основная идея работы [14] состоит в анализе свойств стробоскопических отсчетов $x_{n}=x(n T), v_{n}=v_{x}(n T)$, $y_{n}=y(n T), w_{n}=v_{y}(n T)$ и знакопеременных стробоскопических отсчетов $\tilde{x}_{n}=(-1)^{n} x(n T), \tilde{v}_{n}=(-1)^{n} v_{x}(n T)$, $\tilde{y}_{n}=(-1)^{n} y(n T), \tilde{w}_{n}=(-1)^{n} v_{y}(n T)$ координат и скоростей ионов (где $n$ - номер стробоскопического отсчета), с последующим переносом свойств стробоскопических отсчетов на свойства континуального движения $x(t), y(t)$. Нормированные уравнения движения в электрическом поле (1) с косинусоидальными радиочастотными напряжениями имеют вид

$$
\begin{aligned}
& \frac{d^{2} x}{d \xi^{2}}+\left(a+2 q \cos \left(2 \xi+\varphi_{0}\right)\right) x=0, \\
& \frac{d^{2} y}{d \xi^{2}}-\left(a+2 q \cos \left(2 \xi+\varphi_{0}\right)\right) y=0,
\end{aligned}
$$

где $\xi=\Omega t / 2-$ безразмерное время, $a=8 e U_{0} / m \Omega^{2} r_{0}^{2}$ и $q=4 e V_{0} / m \Omega^{2} r_{0}^{2}-$ безразмерные параметры, периодическая функция $f(\xi)=\cos \left(2 \xi+\varphi_{0}\right)$ имеет безразмерный период $T^{\prime}=\pi$ (безразмерную круговую частоту $\left.\Omega^{\prime}=1\right), \varphi_{0}-$ фаза радиочастотного напряжения в момент старта иона. Для стробоскопических отсчетов $x_{n}=x\left(n T^{\prime}\right), v_{n}=v\left(n T^{\prime}\right), y_{n}=y\left(n T^{\prime}\right)$, $w_{n}=w\left(n T^{\prime}\right)$ справедливы матричные рекуррентные соотношения [23]:

$$
\begin{aligned}
& \left(\begin{array}{l}
x_{n} \\
v_{n}
\end{array}\right)=M_{x}\left(\begin{array}{l}
x_{n-1} \\
v_{n-1}
\end{array}\right)=M_{x}^{2}\left(\begin{array}{l}
x_{n-2} \\
v_{n-2}
\end{array}\right)=\cdots=M_{x}^{n}\left(\begin{array}{l}
x_{0} \\
v_{0}
\end{array}\right), \\
& \left(\begin{array}{c}
y_{n} \\
w_{n}
\end{array}\right)=M_{y}\left(\begin{array}{l}
y_{n-1} \\
w_{n-1}
\end{array}\right)=M_{y}^{2}\left(\begin{array}{l}
y_{n-2} \\
y_{n-2}
\end{array}\right)=\cdots=M_{y}^{n}\left(\begin{array}{c}
y_{0} \\
w_{0}
\end{array}\right),
\end{aligned}
$$

где $v(\xi)=d x(\xi) / d \xi$ и $w(\xi)=d y(\xi) / d \xi-$ безразмерные скорости, $\left(x_{0}, v_{0}\right)$ и $\left(y_{0}, w_{0}\right)-$ начальные условия для уравнений (2) и (3), $M_{x}, M_{y}$ - матрицы монодромии $[22,23]$ для уравнений (2) и (3), удовлетворяющие линейным соотношениям:

$$
\begin{gathered}
\left(\begin{array}{l}
x\left(T^{\prime}\right) \\
v\left(T^{\prime}\right)
\end{array}\right)=\left(\begin{array}{ll}
m_{x x} & m_{x v} \\
m_{v x} & m_{v v}
\end{array}\right)\left(\begin{array}{l}
x(0) \\
v(0)
\end{array}\right)=M_{x}\left(\begin{array}{l}
x_{0} \\
v_{0}
\end{array}\right), \\
\left(\begin{array}{c}
y\left(T^{\prime}\right) \\
w\left(T^{\prime}\right)
\end{array}\right)=\left(\begin{array}{ll}
m_{y y} & m_{y w} \\
m_{w y} & m_{w w}
\end{array}\right)\left(\begin{array}{c}
y(0) \\
w(0)
\end{array}\right)=M_{y}\left(\begin{array}{l}
y_{0} \\
x_{0}
\end{array}\right) .
\end{gathered}
$$

Коэффициенты матриц монодромии $N M_{x}, M_{y}$ подчиняются условиям $m_{x x} m_{v v}-m_{x v} m_{v x}=1, \quad m_{y y} m_{w w}-$ $-m_{y w} m_{w y}=1$ (вследствие сохранения фазового объема). Кроме того, если имеет место устойчивость движения ионов в радиочастотном электрическом поле, должны быть выполнены необходимые и достаточные условия устойчивости $-2<m_{x x}+m_{v v}<2,-2<m_{x}+m_{v v}<2$ [24].
Из условий устойчивости следует параметризация $m_{x x}+m_{v v}=2 \cos \pi \beta_{x}, m_{y y}+m_{w w}=2 \cos \pi \beta_{y}$, где коэффициенты $\beta_{x}, \beta_{y}$ имеют смысл параметров Флоке (секулярных частот), описывающих движение иона вдоль координат $x$ и $y[22,23]$. Из формул (4), (5) получаются рекуррентные соотношения

$$
\left\{\begin{array} { l } 
{ x _ { n + 1 } = m _ { x x } x _ { n } + m _ { x v } v _ { n } , } \\
{ v _ { n + 1 } = m _ { v x } x _ { n } + m _ { v v } v _ { n } , }
\end{array} \quad \left\{\begin{array}{l}
y_{n+1}=m_{y y} y_{n}+m_{y w} w_{n}, \\
w_{n+1}=m_{w y} y_{n}+m_{w w} w_{n},
\end{array}\right.\right.
$$

которые однозначным образом определяют всю цепочку стробоскопических отсчетов по начальным данным. Для стробоскопических отсчетов $x_{n}=x\left(n T^{\prime}\right), v_{n}=v\left(n T^{\prime}\right)$, $y_{n}=y\left(n T^{\prime}\right), \quad w_{n}=w\left(n T^{\prime}\right)$ из формул (6) получаются конечно-разностные соотношения

$$
\begin{gathered}
v_{n}=\left(x_{n+1}-m_{x x} x_{n}\right) / m_{x v}, \quad x_{n+1}-2 \cos \pi \beta_{x} x_{n}+x_{n-1}=0, \\
w_{n}=\left(y_{n+1}-m_{y y} y_{n}\right) / m_{y w}, \quad y_{n+1}-2 \cos \pi \beta_{y} y_{n}+y_{n-1}=0 .
\end{gathered}
$$

Соответственно, знакопеременные стробоскопические отсчеты $\quad \tilde{x}_{n}=(-1)^{n} x\left(n T^{\prime}\right), \quad \tilde{v}_{n}=(-1)^{n} v\left(n T^{\prime}\right), \quad \tilde{y}_{n}=$ $=(-1)^{n} y\left(n T^{\prime}\right), \tilde{w}_{n}=(-1)^{n} w\left(n T^{\prime}\right)$ подчиняются конечно-разностным соотношениям

$$
\tilde{v}_{n}=-\left(\tilde{x}_{n+1}-m_{x x} \tilde{x}_{n}\right) / m_{x v}, \quad \tilde{x}_{n+1}+2 \cos \pi \beta_{x} \tilde{x}_{n}+\tilde{x}_{n-1}=0,
$$

$\tilde{w}_{n}=-\left(\tilde{y}_{n+1}+m_{y y} \tilde{y}_{n}\right) / m_{y w}, \tilde{y}_{n+1}+2 \cos \pi \beta_{y} \tilde{y}_{n}+\tilde{y}_{n-1}=0$,

где $m_{x x}, m_{x v}, m_{v x}, m_{v v}$ и $m_{y y}, m_{y w}, m_{w y}, m_{w w}$ - коэффициенты матриц монодромии $M_{x}, M_{y}$. В [14] считается, что все вычисления должны выполняться в окрестности вершины диаграммы устойчивости $a_{c}=0.236993$, $q_{c}=0.705996 \quad$ в предположении, что $\beta_{x} \approx 1$, $\beta_{y} \approx 0, \quad m_{x x} \approx-1, \quad m_{v v} \approx-1, \quad m_{y y} \approx+1, \quad m_{w w} \approx+1$, $m_{x v} \approx \Pi_{x}=3.7502787, m_{y w} \approx \Pi_{y}=6.8213726, m_{v x} \approx 0$, $m_{w y} \approx 0$, причем при описании движения по координате $x$ нужно использовать лишь уравнения (9), а при описании движения по координате $x y$ лишь уравнения (8). Однако при внимательном анализе можно убедиться, что формулы (7)-(10) сохраняют работоспособность также и в общем случае. В результате как движение по координате $x$, так и движение по координате $y$ можно в зависимости от предпочтений исследователя в любой точке диаграммы устойчивости описывать как обычными стробоскопическими выборками $\left(x_{n}, v_{n}\right),\left(y_{n}, w_{n}\right)$, так и знакопеременными стробоскопическими выборками $\left(\tilde{x}_{n}, \tilde{v}_{n}\right), \quad\left(\tilde{y}_{n}, \tilde{w}_{n}\right)$. Эти способы описания движения ионов будут полностью эквивалентны друг другу.

Представление Флоке $[21,22]$ для решений линейных дифференциальных уравнений (2), (3) с периодическими коэффициентами дает формулы

$$
\begin{gathered}
x(\xi)=g(\xi)\left(C_{1} \exp \left(+i \beta_{x} \xi\right)+C_{2} \exp \left(-i \beta_{x} \xi\right)\right), \\
y(\xi)=h(\xi)\left(S_{1} \exp \left(+i \beta_{y} \xi\right)+S_{2} \exp \left(-i \beta_{y} \xi\right)\right),
\end{gathered}
$$

где в режиме устойчивости параметры $\beta_{x}, \beta_{y}-$ вещественные константы (безразмерные секулярные 
частоты), $C_{1}, C_{2}, S_{1}, S_{2}$ - свободные константы (вообще говоря, комплексные), задающие начальные условия при $\xi=0, g(\xi), h(\xi)$ - периодические функции с безразмерным периодом $T^{\prime}=\pi$, совпадающим с периодом коэффициентов линейных дифференциальных уравнений (2), (3).

В соответствии с (11) для стробоскопических отсчетов $x_{n}=x\left(\xi_{n}\right)$, вычисляемых в точках $\xi_{n}=n T^{\prime}$, справедлива точная формула

$$
x_{n}=x(n)=C_{1} \exp \left(+i \pi \beta_{x} n\right)+C_{2} \exp \left(-i \pi \beta_{x} n\right)
$$

(без ограничения общности можно считать, что нормировка периодической функции-множителя $g(\xi)$ в (11) выбирается так, что $\left.g(0)=g\left(n T^{\prime}\right)=1\right)$. То, что формула (13) удовлетворяет рекуррентным соотношениям (7), можно убедиться прямой подстановкой. Формулу (13) также можно получить, решая однородное линейное конечно-разностное уравнение (7) с постоянными коэффициентами с помощью методов, описанных в [25].

Синусоидальная функция (13), гладким образом интерполирующая стробоскопические отсчеты $x_{n}$ для нецелочисленных значений параметра $n$, совпадает со стробоскопическими отсчетами $x_{n}$ при целочисленных $n$. Очевидно, что эта функция будет тождественно совпадать с общим решением дифференциального уравнения

$$
\frac{d^{2} x(n)}{d n^{2}}+\pi^{2} \beta_{x}^{2} x(n)=0 .
$$

Для знакопеременных стробоскопических отсчетов $\tilde{x}_{n}=\exp (i \pi n) x_{n} \quad$ интерполирующая синусоидальная функция имеет вид

$\tilde{x}_{n}=\tilde{x}(n)=C_{1} \exp (-i \pi(1-\beta) n)+C_{2} \exp (+i \pi(1-\beta) n)$,

при этом функция (15) будет общим решением дифференциального уравнения

$$
\frac{d^{2} \tilde{x}(n)}{d n^{2}}+\pi^{2}\left(1-\beta_{x}\right)^{2} \tilde{x}(n)=0 .
$$

При целочисленных значениях параметра $n$ значения континуальных функций $x(n)$ и $\tilde{x}(n)$ в точности совпадают с дискретными стробоскопическими отсчетами $x_{n}$ и $\tilde{x}_{n}$.

Точно так же из формулы Флоке (12) выводятся синусоидальные функции, интерполирующие стробоскопические отсчеты для координаты $y$. Эти функции являются решениями континуальных дифференциальных уравнений

$$
\begin{gathered}
\frac{d^{2} y(n)}{d n^{2}}+\pi^{2} \beta_{y}^{2} y(n)=0, \\
\frac{d^{2} \tilde{y}(n)}{d n^{2}}+\pi^{2}\left(1-\beta_{y}\right)^{2} \tilde{y}(n)=0 .
\end{gathered}
$$

При целочисленных значениях параметра $n$ значения континуальных функций $y(n)$ и $\tilde{y}(n)$ в точности совпадают с дискретными стробоскопическими отсчетами $y_{n}$ и $\tilde{y}_{n}$.
Уравнения (14), (16)-(18) являются точными способами описания стробоскопических отсчетов (поскольку решения этих уравнений в точках $\xi=n T^{\prime}$ идентичны стробоскопическим отсчетам) для любой точки внутри зоны устойчивости. Сравним уравнения (14), (16)-(18) с уравнениями

$$
\begin{aligned}
& \frac{d^{2} y(n)}{d n^{2}}+4 \sin ^{2} \frac{\pi \beta_{y}}{2} y(n)=0, \\
& \frac{d^{2} \tilde{x}(n)}{d n^{2}}+4 \cos ^{2} \frac{\pi \beta_{x}}{2} \tilde{x}(n)=0,
\end{aligned}
$$

которые получены в [14] для $\beta_{x} \approx 1, \beta_{y} \approx 0$. Из этого сравнения следует, что сделанный в [14] вывод о том, что для стробоскопических выборок безразмерная секулярная частота должна вычисляться по нелинейной формуле $\omega_{s} \approx(2 / \pi) \sin \pi \beta / 2$, а частота биений для стробоскопических выборок должна вычисляться по нелинейной формуле $\omega_{b} \approx(2 / \pi) \cos \pi \beta / 2$ (здесь учитывается переход от номеров стробоскопических отсчетов $n$ к безразмерному времени $\xi=\pi n)$ - это не вполне точный результат, который является следствием приближенного способа получения континуальных уравнений (19) и (20).

Разница между формулами (17) и (19) для секулярных частот, а также между формулами (16) и (20) для частот биений заключается в кубических поправках относительно малого параметра, описывающего отклонение рабочей точки от вершины диаграммы устойчивости. В рамках задачи, исследуемой в [14], эта разница представляется незначительной. Однако расширение области применимости формул (19), (20) на всю зону устойчивости радиочастотного квадруполя будет неправомочной операцией.

Расхождение между приближенными формулами [14] и точными формулами (14), (16)-(18) связано в первую очередь с тем, что замена конечно-разностных соотношений на дифференциальные соотношения с „мгновенными“ производными, которое используется в [14], по самой своей природе является эмпирической и не слишком хорошо обоснованной процедурой. Использование этого метода может приводить к неприятным глобальным расхождениям между предположительно эквивалентными моделями, как это показано на конкретных примерах в [26]. Следует отметить, что подобного рода неприятности не являются редкими при использовании приближенных, качественных или „интуитивно очевидных“ соображений при построении и анализе математических моделей для физических процессов [27-30].

Из точных уравнений (14), (16)-(18) следует, что в любой точке зоны устойчивости безразмерная секулярная частота стробоскопических выборок равна $\beta$, а безразмерная частота биений стробоскопических выборок равна $1-\beta$, где $\beta$ - это параметр Флоке. Надо отметить, что данный результат находится в согласии с аналогичным результатом для континуального движения ионов $[19,23]$. Тем самым при описании движения ионов 
в радиочастотных квадрупольных полях нет оснований для введения специфических секулярных частот и соответствующих им специфических эффективных потенциалов для стробоскопических отсчетов координат и скоростей, как это делается в [14]. Альтернативная теория [19] для квадратичного эффективного потенциала в радиочастотных квадрупольных полях, которая основана на матричном представлении Флоке-Ляпунова [22], приводит к точно такому же выводу.

Использование формул [19] позволяет получить в окрестности вершины зоны устойчивости $a_{c}=0.236994$, $q_{c}=0.705996$ при малых вариациях $\delta a=a-a_{c}$ и $\delta q=q-q_{c}$ рабочих параметров $a, q$ следующие оценки для секулярных частот и для минимаксного (не зависящего от начальной фазы радиочастотного напряжения) аксептанса квадруполя, где для коэффициентов сохранены три знака после запятой:

$$
\begin{aligned}
\beta_{x} \approx 1.000-0.716 \sqrt{-\delta a-1.154 \delta q}, \\
\bar{V}_{x}^{2} \approx r_{0}^{2} \frac{(-\delta a-1.154 \delta q)}{2.783}, \\
\bar{R}_{x}^{2} \approx r_{0}^{2} \frac{(-\delta a-1.154 \delta q)}{0.378}, \\
\beta_{y} \approx 1.121 \sqrt{\delta a-0.640 \delta q}, \\
\bar{V}_{y}^{2} \approx r_{0}^{2} \frac{(\delta a-0.640 \delta q)}{3.749}, \\
\bar{R}_{y}^{2} \approx r_{0}^{2} \frac{(-\delta a-1.154 \delta q)}{0.218} .
\end{aligned}
$$

Технические детали проделанных вычислений содержатся в $[31,32]$.

В приведенных формулах $(21)-(26)$ величины $\beta_{x}, \beta_{y}$ - безразмерные секулярные частоты в окрестности вершины зоны устойчивости для координатных осей $x$ и $y ; \bar{V}_{x}^{2}, \bar{V}_{y}^{2}$ - параметры, имеющие физический смысл квадрата нормированной скорости и характеризующие глубину псевдопотенциальных ям по $x$ и $y$ (максимальную начальную кинетическую энергию иона, при которой ион, стартуя с оси квадруполя, удерживается электрическим полем внутри межэлектродного промежутка $\left.\pm r_{0}\right) ; \bar{R}_{x}^{2}, \bar{R}_{y}^{2}$ - параметры, имеющие физический смысл квадрата расстояния и характеризующие ширину псевдопотенциальных ям по $x$ и $y$ (максимальное начальное смещение иона с оси квадруполя, при которой ион, стартуя с нулевой начальной скоростью, удерживается электрическим полем внутри межэлектродного промежутка $\left.\pm r_{0}\right)$. Чтобы рабочая точка оставалась в пределах зоны устойчивости, должны быть выполнены приближенные неравенства $-\delta a-1.154 \delta q>0, \delta a-0.640 \delta q>0$. Полный или минимаксный аксептанс (множество начальных условий $\left(x_{0}, v_{0}\right)$ и $\left(y_{0}, w_{0}\right)$, при которых ион удерживается электрическим полем внутри межэлектродного промежутка $\pm r_{0}$ независимо от начальной фазы радиочастотного поля) с хорошей точностью описывается эллипсами фазового пространства [19]:

$$
\frac{v_{0}^{2}}{\bar{V}_{x}^{2}}+\frac{x_{0}^{2}}{\bar{R}_{x}^{2}} \leq 1, \quad \frac{w_{0}^{2}}{\bar{V}_{y}^{2}}+\frac{y_{0}^{2}}{\bar{R}_{y}^{2}} \leq 1 .
$$

Сравнение выражений (22) и (25), а также результатов численного моделирования траекторий ионов с оценками глубины псевдопотенциальных ям в [14] показывает удовлетворительное совпадение этих результатов при малых отклонениях $0 \leq \beta_{y} \leq 0.1$ и $0 \leq 1-\beta_{x} \leq 0.1$, когда рабочая точка $(a, q)$ находится в окрестности вершины зоны устойчивости радиочастотного квадруполя.

Отметим еще одно важное отличие в полученных результатах. В работе [14] при оценке полного (минимаксного) аксептанса (27) линейного квадруполя в качестве ширины псевдопотенциальной ямы используется размер межэлектродной диафрагмы $r_{0}$. В действительности (см. [16,19]), ширина псевдопотенциальной ямы стремится к нулю при приближении к точкам $(a, q)$ на границах диаграммы устойчивости (за исключением вырожденной точки $a=0, q=0$ ) в полном соответствии с формулами (23), (26). В конечном итоге это приводит к некорректной оценке полного аксептанса квадруполя в [14], хотя оценка глубины пседопотенциальных ям в [14] при вариации $\beta_{x}, \beta_{y}$ точна с погрешностью порядка кубических поправок по малому параметру отклонения рабочей точки от вершины зоны устойчивости. При этом необходимо подчеркнуть, что идея анализа стробоскопических отсчетов и огибающих стробоскопических отсчетов вместо самих траекторий, предложенная в [14], обладает несомненным методологическим изяществом, а понятие глубины псевдопотенциальной ямы, введенное авторами [14] в самом общем случае без обычного допущения о малости параметра $q$ и без привязки к конкретной модели псевдопотенциала, является важным вкладом в общую теорию радиочастотных квадруполей и заслуживает всяческого внимания.

\section{Благодарности}

Авторы благодарны редакции ЖТФ за возможность продолжить в широком кругу научную дискуссию, начатую в ЖЭТФ публикацией [14]. Авторы благодарны рецензенту настоящей работы за доброжелательный отзыв и важные уточнения, позволившие исправить некоторые технические ошибки и значительно улучшить текст работы.

\section{Финансирование работы}

Работа выполнена в рамках гос. задания № 075-0078019-00 ИАП РАН.

\section{Список литературы}

[1] Paul W., Steinwedel H. // Zeitschrift für Naturforschung. 1953. Vol. A8. N 7. P. 448-450.

[2] Слободенюк Г.И. Квадрупольные масс-спектрометры. М.: Атомиздат, 1974. $272 \mathrm{c.}$

[3] Dawson P.H. Adv. in Electronics and Electron Physics. Vol. 53. Academic Press, 1980. P. 153-208.

[4] March R.E., Hughes R.J. Quadrupole Storage Mass Spectrometry. New York: John Wiley and Sons, 1989. 471 p. 
[5] Dawson P.H. Quadrupole Mass Spectrometry and Its Applications. Woodbury: American Institute of Physics, 1995. $343 \mathrm{p}$.

[6] Major F.G., Gheorghe V.N., Werth G. Charged Particle Traps. Physics and Techniques of Charged Particle Field Confinement. Springer-Verlag, 2005. 355 p.

[7] Werth G., Gheorghe V.N., Major F.G. Charged Particle Traps II. Applications. Springer-Verlag, 2009. 276 p.

[8] Мак-Лахлан Н.В. Теория и приложения функций Матье. М.: ИИЛ, 1953. 476 с.

[9] Ландау Л.Д., Лифиии Е.М. Механика. Сер. Теоретическая физика. Т. І). М.: Физматгиз, 1958. С. 119-123.

[10] Гапонов А.В., Миллер М.А. // ЖЭТФ. 1958. Т. 34. С. $242-$ 243.

[11] Gerlich D. In: Adv. in Chemical Physics Series, vol. LXXXII / Ed. by C.-Y. Ng, M. Baer. NY.: John Wiley \& Sons, 1992. P. 1197.

[12] Sudakov M. // Int. J. Mass Spectrom. 2001. Vol. 206. P. $27-43$.

[13] Baranov V.I., Bandura D.R., Tanner S.D. // Int. J. Mass Spectrom. 2005. Vol. 247. P. 40-47.

[14] Судаков М.Ю., Апацкая М.В. // ЖЭТФ. 2012. Т. 142. С. $1-8$.

[15] Gao C., Douglas D.J. // J. Am. Soc. Mass Spectrom. 2013. Vol. 24. P. $1848-1852$.

[16] Douglas D.J., Berdnikov A.S., Konenkov N.V. // Int. J. Mass Spectrom. 2015. Vol. 377. P. 345-354.

[17] Reilly P.T.A., Brabeck G.F. // Int. J. Mass Spectrom. 2015. Vol. 392. P. 86-90.

[18] Brabeck G.F., Reilly P.T.A. // J. Am. Soc. Mass Spectrom. 2016. Vol. 27. P. 1122-1127.

[19] Berdnikov A.S., Douglas D.J., Konenkov N.V. // Int. J. Mass Spectrom. 2017. Vol. 421. P. 204-223.

[20] Бердников А.С., Галль Л.Н., Галль Н.Р., Соловьев К.В. // Научно-технич. ведомости СПбГПУ. Физ. мат. науки. 2018. T. 11. № 3. C. 52-64.

[21] Floquet G. // Ann. Sci. de l’École Norm. Sup. 1883. Vol. 12. P. 47-88.

[22] Демидович Б.П. Лекции по математической теории устойчивости. М.: Наука, 1967. 472 с.

[23] Konenkov N.V., Sudakov M., Douglas D.J. // J. Am. Soc. Mass Spectrom. 2002. Vol. 13. P. 597-613.

[24] Verentchikov A., Berdnikov A., Yavor M. // Physics Procedia. 2008. Vol. 1. P. 87-97.

[25] Гельфонд А.О. Исчисление конечных разностей. Изд. 2-е доп. М.: ГИФМЛ, 1959. 400 с.

[26] Бердников А.С., Веренчиков А.Н., Коненков Н.В. // Массспектрометрия. 2017. Т. 14. С. 176-189.

[27] Буляница А.Л., Курочкин В.Е. // Научное приборостроение. 2000. Т. 10. № 2. С. 43-49.

[28] Евстрапов А.А., Буляница А.Л., Рудницкая Г.Е., Беленький Б.Г., Петряков А.О., Курочкин В.Е. // Научное приборостроение. 2003. Т. 13. № 2. С. 57-63.

[29] Буляница А.Л., Евстрапов А.А., Рудницкая Г.Е. // Научное приборостроение. 2003. Т. 13. № 4. С. 28-40.

[30] Буляница А.Л. // Научное приборостроение. 2005. Т. 15. № 2. C. $51-66$.

[31] Бердников А.С., Кузьмин А.Г., Масюкевич С.В. // Научное приборостроение. 2018. Т. 28. № 3. С. 90-100.

[32] Бердников А.С., Кузьмин А.Г., Масюкевич С.В. // Научное приборостроение. 2018. Т. 28. № 4. С. 135-145. 